\title{
Approaching to Death: A Study About Dream Contents and Self-images in Hospice
}

\author{
Vincenzo Caroprese \\ Hospice at Local Health Service, Lecce, Italy
}

\author{
Maria Rita Serio, Antonio Godino \\ University of Salento, Lecce, Italy
}

\begin{abstract}
This research is focused on a large sample of patients in a specialized ward for palliative therapies of 12 bed-places (hospice), aiming at the treatment of pain and psychological disorders in patients in terminal state of their disease (mainly cancer). The aim of the research is to find a rapport among antalgic (FANS (Farmaci Antiinfiammatori Non Steroidei) - NSAID (non-steroidal anti-inflammatory drugs)) and opiate therapies protocols, emotional and affective changes, structures and contents of dreams in both self-report and as recalled during a semi-structured interview. A total of 34 subjects, ranging from 32 to 84 years old, with a clear and awake consciousness state, all studied with multi-testing for pain scaling: Visual Analogic Scale; Verbal Numerical Scale; measure PQRST (provokes/alleviates, quality, irradiation, severity, time (continuous, discontinuous, nocturnal, and diurnal)), together with dream-content diary self-report at wake-up and clinical interview (averaging nearly $3 /$ ss, up to a total of 97 interviews) by which patients recall various qualities of their dreaming experiences (affective tone, density, and speed of changes) and describe main dream contents (identities, characters, backgrounds, recurrent themes in the stories, realism vs. symbolism). There is little difference, in rapport with age, gender, and antalgic treatment with FANS-NSAID, as for the number of dream, but age and male gender are associated with an important average number increase when opiate treatment is begun or raised. Themes can be categorized within a twofold type: type A - mainly symbolic, or type B - mainly realistic and obsessive. There is a shift towards type B or mixed relating to age (elders) and opiate treatment increasing. The affective quality of dreams is less threatening, mainly in elders of male gender, in association with opiate treatment beginning and/or increase. As opiate does control the affective components of pain only, promoting both REM (rapid eye movements) phase sleeping and emotional detachment, results in dream contents and affective qualities are to be expected, but further research is needed as for differences associated with age and gender variables.
\end{abstract}

Keywords: hospice, dream-contents, opiates, pain control, affective shifts

\section{Introduction}

The patients admitted in the hospice are subjects experiencing a really singular condition: They are not hospitalized in view to cure or recover from their illness, as there is no more hope to be able to do something effective in the fight against it, but simply to be attended and treated against the pain and psychological discomfort during the short amount of time left before death.

They are approaching to death, as their prognostic is clear-cut and explicit by physicians, and they are

Vincenzo Caroprese, director, Hospice at Local Health Service.

Maria Rita Serio, Ph.D., professor, University of Salento.

Antonio Godino, Ph.D., full professor, University of Salento. 
fully aware of this outcome. These patients are anymore defending themselves with deceptive illusions and hopes of gaining the battle against the cancer: All they need now is not to be degraded by pain, to feel alone, or to quietly prepare for their passing and the end of life (Godino \& Serio, 2002; Kübler-Ross, 1986).

Terminally ill patients are an interesting natural experiment with some controlled variables, such as awareness, normal level of consciousness as they are not being in coma, a common and unique prognosis, palliative treatment, to permit a demonstrative study of some psychological changes, and in our research, to study in particular the rapport between pain control and the phenomenology of dreams (Godino \& Serio, 2007; Toscani, 1997).

Pain has two main components: a sensory-perceptive and an affective one. Many analgesic drugs (in particular, opiates) do not have anesthetic activity (that is to say, they do not modify nor stop transmission of sensorial signals from receptors toward the brain) at all, and the patient reports that he/she continues to perceive the same physical inputs, yet he/she does not feel pain any more.

The dissociation between affective and perceptive-sensory domains can be obtained, beyond and together with drugs, also with techniques of psychological kind. Among these techniques, we must remember, in particular, the hypnosis and several techniques of relaxation and conditioning, like the yoga, the autogenic training standard-type, and the respiratory autogenic training. Their usefulness is very well verified in the preparation to the delivery, in the dental analgesia, in supporting the pain deriving from the intense and extended effort of agonistic sport activities, and in order to contrast the pain usually associated to invasive medical examinations (Godino \& Toscano, 2007).

In these cases, we still speak about "simple" or elementary pains, in which a single factor can be isolated, as being main or exclusive. In many other cases, the pain perception is an aspect, certainly important and overwhelming but not central, of an uneasiness with many dimensions. Surely, this is the case of the pain in the sick one in the terminal phase. The study of the disease in advanced and terminal phase is clearly a multidimensional one; the various aspects, organic or psychological, are deeply interlaced from each other and are lived with much intensity by the sick one, the relatives, and the sanitary staff. Generally, the organic aspects are focused and treated in the first place, while the psychological dimension is left behind as a secondary problem, like a simple by-effect of the disease. This strategy of work risks to prevent the understanding of psychological and relational dynamics that follow the disease, as it is the case of the frequent link between depressive reactions and pain (Romano \& Turner, 1985).

In the light of what has been stated in these lines, it appears obvious, alike, that the psychological competence is important: (1) for being able to pick operating dynamics in various situations and contexts; and (2) to the aim to favor the formation of more adaptive attitudes and working styles.

The task of the psychologist, moreover, is aimed to develop, both within assistance team and the families, the abilities to know how to contain and elaborate tensions and suffering in the possible better way. The psychological participation can be realized in two directions: on one side, working towards the sanitary team; on the other side, working directly with the patients and their families. Within a more complex study on psychological prevention of affective disturbances in the patients and their relatives and burn-out in the sanitary staff of the hospice, we focused our search here on the rapport of pain treatment with opiates and the changes in contents and emotional qualities of reported and recalled dreams of terminal patients (Burish, 1995; Tiengo, 2004; Toscani, 1997). 


\section{Subjects and Method}

Subjects are all patients attended in a hospice (a specialized hospital ward for palliative treatments) with a total of 12 single rooms. Every subject has been studied both with self-report on pain and multiple interviews (averaging three in a period of 21 days) centered on affective condition and content of dreams.

Testing of pain was made using Osgood type scales, like a Visual Analogic Scale to be marked every 4-6 hours during day time (or at least three times a day) in a format alike the one in Figure 1.

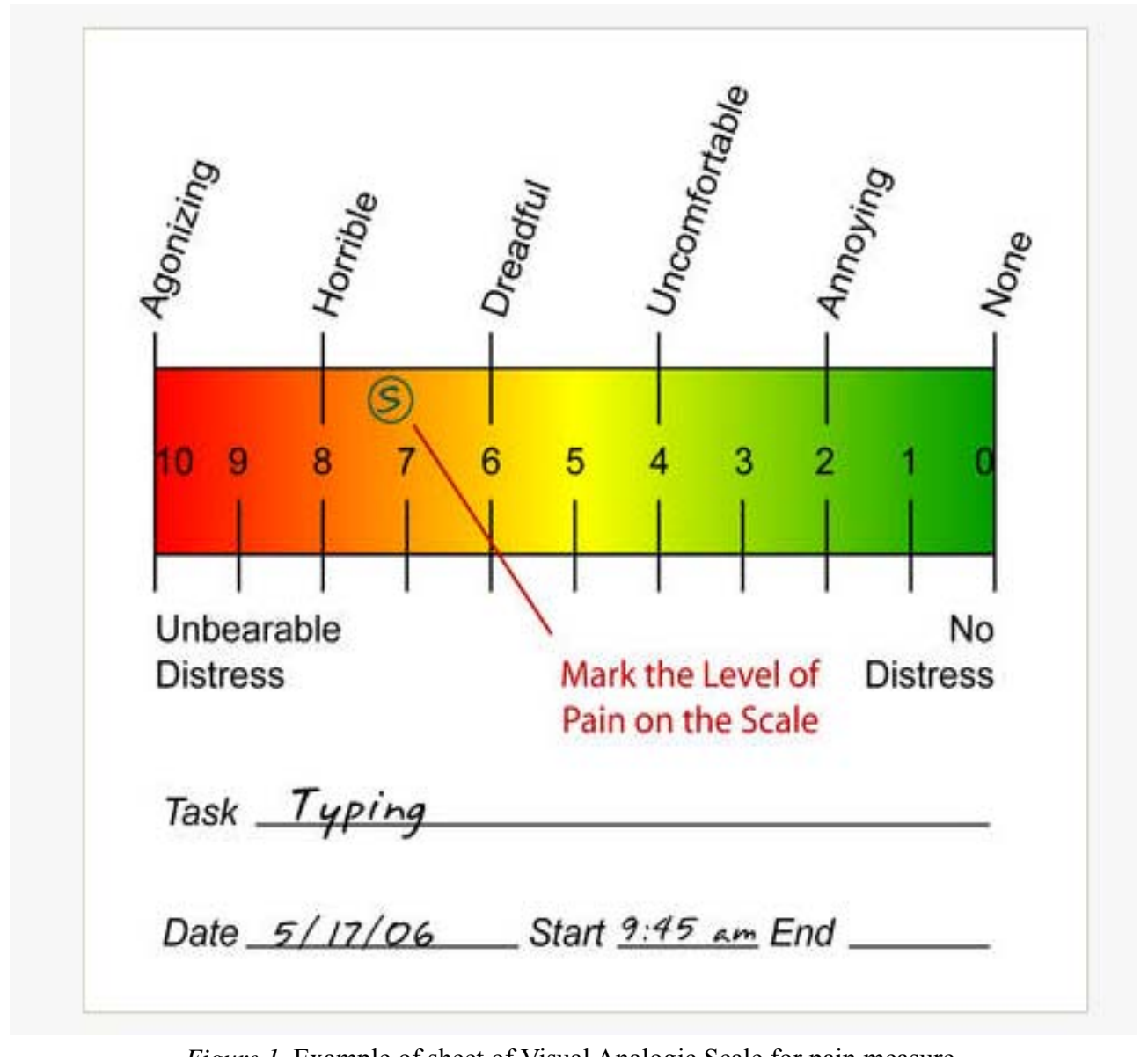

Figure 1. Example of sheet of Visual Analogic Scale for pain measure.

Patients were also asked to take a pain journal using a pain numerical scale and with some advices like these about what to consider and mark:

Pain descriptor words - Is your pain burning? Tingling? Pulsating?

Using pain descriptor words in your journal can help you track changes and patterns in your pain quality. It can also help doctors pinpoint your type of pain.

Time of day-Do you hurt more in the morning or the evening?

How are your afternoons?

What you are doing when your pain begins - Did you just get out of bed, or had you been sitting for awhile when your pain started? Write down how you feel after activities, such as walking, reading, eating, washing, etc..

Pain scale rating-Most pain scales use the $0-10$ rating system, with 0 representing no pain and 10 representing the worst imaginable pain.

Your pain will usually fall somewhere in between. 
The pain journal technique is a tool of valuable interest not only for research issue, but also for improving the patient's condition of chronic pain, in order to gain control of it (Guarino, 2010). The study comprises a synchronous monitoring (blood cortisol incretion level isochronal at morning, blood immunoglobuline level) of the biological pointers of modulation of immunity responses to stressors and a standardized examination of the verbal and nonverbal exchanges, of the relation between styles of facing of the problems (negation, aggressiveness, passive folding, etc.) and evolution of the pain. Observations comprise both the "organic" side of the clinical picture (immunity decrease connected to the reaction of pathogenic stress) and psychiatric clinical picture (depressive reaction, yielding of the sense of integrity and self-esteem, etc.). The examination is made with an "ad hoc" psychiatric interview (with 10 items with forced answers and 10 items with open-ended answers), plus an observable protocol (temporized and segmented) of the communications coming from the team toward the patient and his/her relatives.

Both the psychological interview and the diary comprise an examination and recall of the sleeping outlook (eventual initial or terminal insomnia, deepness, quality, and content of sleeping, etc.) together with a diary and a recall of dream contents and qualities.

Drugs used to treat pain were, mainly, associations of FANS (Farmaci Antiinfiammatori Non Steroidei) - NSAID (non-steroidal anti-inflammatory drugs)) and slow absorption opiates (retard). The side effects of antalgic drugs commonly comprise sleepiness, respiratory (tachypnea, shortness of breath), and cardiological (tachycardia at rest, lowered inotropism) symptoms, that are disturbing for the patients but can also be very dangerous in view of their critical medical condition (Plonk \& Arnold, 2005; Le Grand \&Walsh, 1999).

We must remember that an un-balanced use of opiates can be lethal and could be a factor of anticipation of death, like in euthanasia (World Health Organization, 1995).

Another side effect of opiates is the preservation of the REM phase of sleep, that can be reached more quickly and lasts longer (Engstrom, 1999; Mercadante \& Ripamonti, 2002).

The recall of dreams was made in part by the patients in their diaries and to a much greater extent within the psychiatric interviews. Questions about dream experiences were focused both on subjective qualities (for instance, pleasant/unpleasant, quick changes in actions/slowness or stillness, etc.) and contents (for instance, identity of the characters, gender, type of actions, ambience and background, etc.).

The total number of subjects studied is 34 , for a total of 97 interviews, with an age ranging from 32 to 84 (median $=72$ ), chosen to balance gender (17 males and 17 females) as the hospice inmates are mainly $(60 \%-40 \%)$ males.

The whole time period of the observational phase of this study has been of nearly seven months.

\section{Results and Discussion}

In our sample, sleep disturbances are very common (as expected, because of breathing symptoms, metastatic pains, and psychological distress) with a prevalence approaching $90 \%$. Nearly all subjects have mild to moderate insomnia, with an average of three sleep disruptions (lasting an average of 40 minutes each one) and early awakening (up to over two hours before the normal awakening time). This clinical situation is not steady as in the beginning, or the increase of opiates is followed by a rapid decrease in number and length of sleep disruptions (averaging two per night and an average length of 20 minutes), and the terminal insomnia becomes less important (early awakening of under an hour before the normal awakening time). 
The total amount of sleeping time increases, after analgesic medication with opiates, from an average of four and half hours up to an average of six and half.

Immune depression is not generally severe, being mild to moderate, but is present in nearly all subjects (32 out of 34) as for blood tests, with signs like cortisol increase and neutropenia, with a clinical situation that is largely diversified (from mild inflammatory signs with joint aches and stiffness, to recurrent fever and infective symptoms with interstitial pneumonias and cystitis).

In nearly $60 \%$ of the sample (23 out of 34), a typical paraneoplastic syndrome is observed (Voltz, 2002).

The report of dreams in the diaries is not reliable, as only $40 \%$ of the journals contain dream narratives, however, after increase or beginning of opiate treatment, the percentage rate of dreams is reported nearly doubles. Nearly all the reports are present only in the morning awakening diary and not within the episodes of sleep disruption. Indeed, the disruptions themselves are not immediately reported in the journal but described later, at the time of the morning awakening.

There is an important difference between the narrative content of the dreams reported in the journal and the dreams recalled in the interviews.

Dreams reported are less coherent, less organized, much more expressive, and richly detailed than the dreams recalled. Dreams recalled are poor in details and fading, but at the same time much more realistic and simple to follow and understand. Altogether, we must reiterate that the comparison is unreliable, as the reported dreams appear to be a very partial selection of actual dreams.

In elders subjects (over 70 years old), the affective tone is mainly of detachment and surrender, while in younger subjects (under 50 years old), the feelings of anger/despair or depressive surrender are prevalent. As for the depression prevalence, interview data do not confirm a clear depressive syndrome diagnosis, also if there are multiple signs of depressed moods (as terminal insomnia, hostility, polarized thoughts, passivity, etc.). With the opiate medication, there is a mild euphoric state of mind as the patients feel freed from pain. The great importance of pain control for gaining a better quality of life in terminal patients and obtaining a net change in mood, what has been observed in large review studies, is also confirmed in this research (Mitchell et al., 2011).

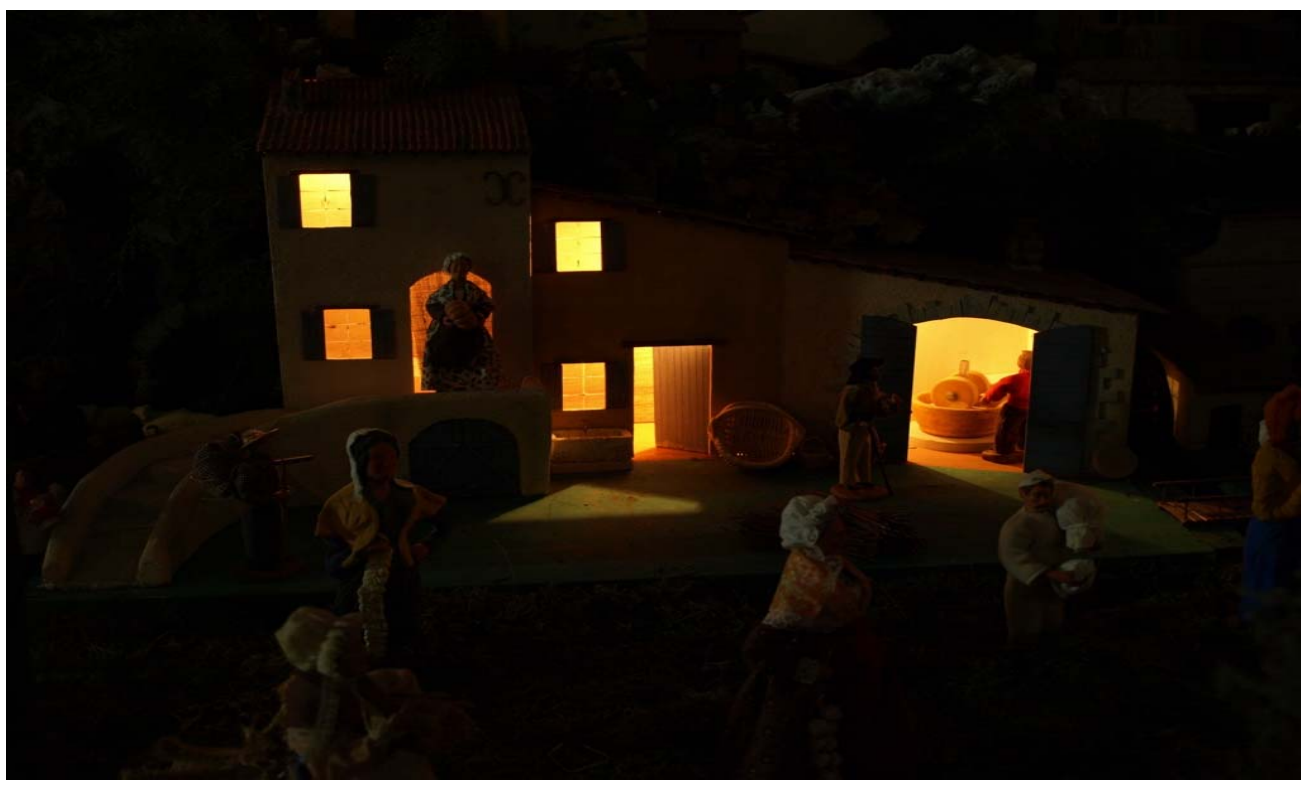

Figure 2. Image resembling the typical high contrast ambiance of many dreams. 
Age, gender, and pain treatment with FANS - NSAID are not related with the total number of dreams reported or recalled, but a significant increase is observed after the beginning or rise of opiate medication. The change is present in all subjects, but is more important in males than females, in elders more than younger. Probably, this is a combined effect of mood change and REM phase facilitation by the opiates. The subjects of male gender and more aged are generally more depressed than other sub-groups, and with more marked insomnia, so the change in mood induced by the medication with opiates appears larger.

Themes of dreams are often recursive, such as to see objects and places under a dramatic and contrasted light, with deep dark and spotted light, somehow resembling the image reconstruction in Figure 2.

Another recurrent theme is to be in a familiar environment, like a house, a town, or a working place, that changes as the subject walks and moves on, until it turns out quite strange and horrible to see passages and streets are absurdly narrow or sloping in a dizzy way, and the dreamer feels confused and scared.

Often, the subject dreams to be doing some activity he/she typically made when he/she was young and healthy, as typing, doing housework, or driving a car, but these activities are abruptly stopped and resumed several times, there is not a clear ending, and the effort is very tiring and useless.

Otherwise, the subject is witness of a car accident, collapses, disasters of water and mud, but all is unreal and without any tragic ending (collapses do not hurt or kill, the dreamer can jump over mud as if it were rubber, the mass of water overwhelms but does not drown, etc.).

The fourth most common theme is to discover (in a lost coin of furniture, luggage, drawers, a pocket of an old coat, etc.) things pertaining to the past life of the dreamer, like objects of the childhood, such as photos, little toys, or pieces of cloth of childish clothes. Sometimes, the photos begin to move and/or speak, the clothes appear animated as if they were puppets, somehow like a dream within the dream.

Dream themes tend to recur in the same subjects, creating a sense of uneasiness, as if they were obsessive and intrusive thoughts, with the subjects feeling trapped and unable to escape.

As for the quality of thematic content, two kinds of symbolic structures are distinguishable:

(1) Type A symbols and themes—Direct symbols, familiar situations turning scaring, images of the past fading away and re-living, life situations confused and under attack, etc.;

(2) Type B symbols and themes - Dreams that appear a symbolic and the direct expression of impotence, inability to cope, frightening associated to images of death, paralysis, etc..

Hither is exposed an example of type B dream (dream reported at awakening):

I am in a house, maybe a hotel, whose stairs are not internal but only external, are in metal, one is forced to climb on the stair as if it were a mountain climbing. To climb is difficult but not much scaring, going downstairs (in order to getting out) is really dangerous and very difficult. At last I get out, and find myself in a narrow street (not in my town, but unfamiliar) with bends, pillars, arcades. Going on, with a great effort, I am higher and higher (alike before in the hotel) but there are no more urban forms, houses, cars, I am on some strange mud hills, incumbent upon the narrow and bended lanes and streets, down there, of the far-away town. I must return, at all costs, because I do not miss my airplane, that is near to take-off. Desperate, I jump down on the hills, bouncing on mud as if it were rubber, I fly and jump at the same time. Yet there are not ways to airport, all streets are blocked, indirect and bended, neither in taxi I could reach my airplane. I know there is no more time left, I awake in panic.

When medication with opiates is increased, there is a shift from type A and mixed types towards type B dreams (see Figure 3). Emotional tonality of dreams becomes less frightening and the number of reported dreams increases. 
The shift toward type B dreams can be understood as a result of a sharpened awareness of the approaching to death combined with the abandonment of indirect symbols and other unconscious tricks to veil and mask this perception.

This change, once again, is more evident in males than in females and in older than in younger subjects.

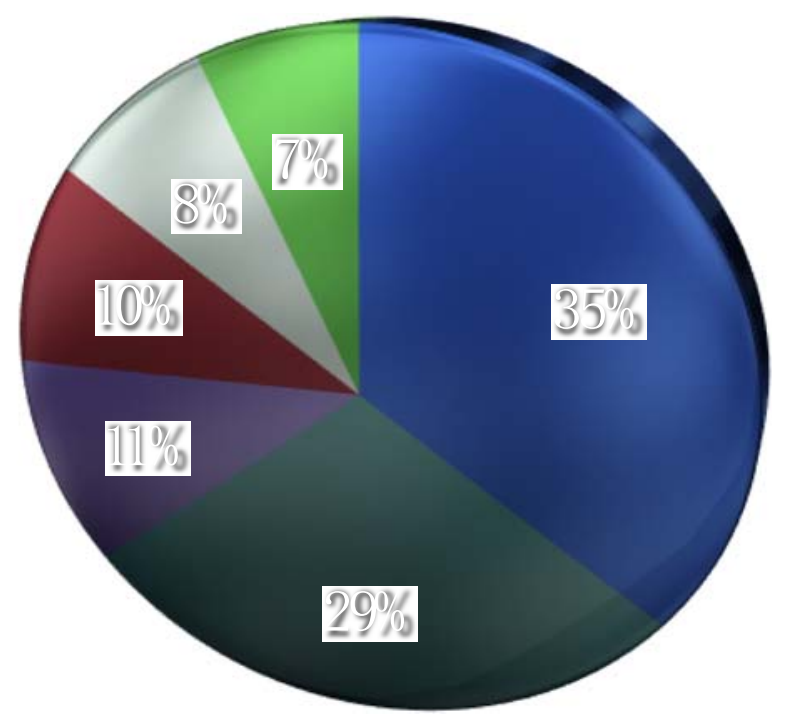

Figure 3. Percentage of type A (35\%) and type B (29\%) dreams, other mixed.

\section{Conclusions}

This study is of phenomenological type, but the sample is highly specific and the variables are sufficiently constant to permit results to be, at least in part, generalized.

Pain control obtained with FANS - NSAID medication does not change the number of dream reports, nor their contents or their affective qualities. Also, the dream recall in interviews stays unchanged.

If the contents of dreams are interpreted as a manner of the self-image to emerge following the rules of the unconscious, it is seen that this emersion is boosted, in large measure in males and older patients, by the beginning or the rise of medication with opiates.

While the main effect of antalgic action of opiates is a change in a depressive mood during awake in day time toward a mild euphoric mood and detachment in rapport with fears and the panic associated to the approaching of death, in sleep time opiates increase the emergence of dreams whose content is more explicit and overt, leaving aside the defense of the symbolic shield. Alongside with this change in contents and structure of dreams, its affective quality is not worsened, as we could expect, but on the contrary feelings are somehow of a witnessing with milder implication and a relative detachment.

The number of dreams, both reported in diaries and recalled in interviews, increases markedly in all subjects. Such change is expected because opiates promote both sleeping time and REM phases, but further research is needed to explain the large differences observed in males vs. females and in older subjects vs. younger ones. Moreover, further research is needed to understand the opiate-induced gap between the increased and clear-cut awareness of approaching to death and the decrease in depressive moods. 


\section{References}

Abiven, M. (2001). To attend the terminal ill. Torino: Centro Scientifico Ed..

Antonelli, F. (1981). In order to die living: Psychology of death. Roma: Città nuova.

Arcuri, E., \& Tirelli, W. (1998). Cancer: Attend the needs of the sick. Roma: Il Pensiero Scientifico.

Barnes, R. (1979). Pain and suffering: A psychosomatic problem. Dyn. Psych., 12, 162.

Burish, T. (1995). Conditioned side effects induced by cancer chemotherapy: Prevention through behavioral treatment. In S. Andrew (Ed.), Psychosocial processes and health. Cambridge: Cambridge Univ. Press.

Campione, F. (1991). Psychological care for terminal sick. In Trattato di psicologia medica. Armando, Roma.

Campione, F. (2000). To live again: Psychological help in crisis situations. Clueb, Bologna.

Campione, F. (2003). The right of the dying person to know the truth. In Assenza più acuta presenza. Paoline, Milano.

Engstrom, C. A. (1999). Sleep alterations in cancer patients. Cancer Nursing, 22, 143-148.

Godino, A., \& Serio, M. R. (2002). The shadow of death: A psychodiagnostic study. Psychofenia, V(8), 41-60.

Godino, A., \& Serio, M. R. (2007). The appeal of pain: The reasons of a research. In Cure palliative e terapia antalgica, highlights nel Salento. Atti, Pensa, Lecce.

Godino, A., \& Toscano, A. (2007). Hypnosis: History and techniques. Franco Angeli, Milano.

Grassi, L., Biondi, M., \& Costantini, A. (2003). Practical handbook of psychological oncology. Roma: Il Pensiero Scientifico Ed..

Grassi, L. (1999). Interactions between cancer and depression. Depressione, 7(2).

Grinberg, L. (1978). Guilt and depression. Il Formichiere, Milano.

Guarino, A. H. (2010). Get your lower back pain under control. New York: John Hopkins University Press.

Hannah, B. (1964). Regression or renewing in the elder. In Psychotherapeutische problem. Rascher, Zurigo.

Kübler-Ross, E. (1986). Death: The final stage of growth. New York: Simon \& Schuster.

Le Grand, S. B., \& Walsh, D. (1999). Palliative management of dyspnea in advanced cancer. Curr Opin Oncol, 11, $250-257$.

Mercadante, S., \& Ripamonti, C. (2002). Medicine and palliating cures in oncology, clinical, aiding, organizational aspects. Masson, Milano.

Mitchell, A. M., Chan, M., Bhatti, H., Halton, M., Grassi, L., Johansen, C., \& Meader, N. (2011). Prevalence of depression, anxiety, and adjustment disorder in oncological, haematological, and palliative-care settings: A meta-analysis of 94 interview-based studies. Lancet Oncol, 12, 160-174.

Plonk, W. M., \& Arnold, R. M. (2005). Terminal care: The last weeks of life. J. Palliat Med., 8(5), 1040-1041.

Raymond, A., Moody, J., \& Dianne, A. (2003). Life after a great sorrow. Armenia, Milano.

Romano, J. M., \& Turner, J. A. (1985). Chronic pain and depression: Does the evidence support a relationship? Psychological Bulletin, 97(1), 18-34.

Serio, M. R. (2004). Near to death. Milella, Lecce.

Smith, R. C., \& Hoppe, R. B. (1991). The patient's story: Integrating the patient and physician-centered approaches to interviewing. Ann. Intern. Med., 115, 470-477.

Spiegel, D., Boolm, J. R., Kraemer, H. C., \& Gotthil, E. (1989). Psychological support for cancer patients. Lancet, II, $1447-1452$.

Tiengo, M. (2004). The pain: An anthropological perspective. MNL, Bologna.

Toscani, F. (1997). The terminal sick. Il Saggiatore, Milano.

Voltz, R. (2002). Paraneoplastic neurological syndromes: An update on diagnosis, pathogenesis and therapy. Lancet Neurol, 1, 294-305.

Von Franz, M. L. (1986). On death and dreams. Bollati Boringheri, Torino.

World Health Organization. (1995). National cancer control programmes: Policies and managerial guideline. Geneva: World Health Organization. 